\title{
Electrochemistry in Deep Eutectic Solvents
}

Chiemela A. Nkuku and Robert J. LeSuer

Chicago State University, Dept. of Chemistry and Physics, Chicago, IL 60628

S-2: Flow induced by the compression of two disks

S-4: Derivation of Pe- $v$ relationship for a non-Newtonian fluid

S-5: Figure S-1. Chronoamperometry of ferrocene in 1:2 ChCl:TFA DES.

S-6: Figure S-2. Simulation of tip-velocity dependent approach curves with varying RG.

S-7: Figure S-3. Comparison of numerical and analytical solutions to the radial fluid velocity profiles.

S-8: Figure S-4. Comparison of numerical and analytical solutions to the maximum radial velocity.

S-9: COMSOL script used to general velocity-dependent approach curves.

Two video files, in AVI format, show the concentration profiles during an approach curve under low and high Pe conditions. The file LOWPE.AVI is a simulation with $\mathrm{Pe}=0.01$ and HIPE.AVI is a simulation with $\mathrm{Pe}=5$. 


\section{Flow induced by the compression of two disks}

[Note that the somewhat unconventional notation is used to minimize confusion with variables used in SECM terminology.]

Two disks of radius $\mathrm{R}$ are separated by a distance $\mathrm{d}$. For convenience, the midpoint between the two plates is set at $z=0$. Fully developed, one dimensional flow can be described as (Bird et al. 2002):

$$
\begin{aligned}
& \frac{1}{z} \frac{d}{d z}\left(\eta z \frac{d u}{d z}\right)=-\frac{\Delta p}{R} \\
& \frac{d u}{d z}(0)=0, u(d / 2)=0
\end{aligned}
$$

where $u$ and $\eta$ are the fluid velocity and viscosity, respectively, and $\Delta p$ is the pressure difference. The intent is to develop equations that describe the maximum and average fluid velocities in the gap between the two disks as they move towards one another at a velocity, $v$. The solution to S.1 is

$$
u(z)=\frac{\Delta p\left(d^{2}-4 z^{2}\right)}{16 R \eta}
$$

A moving disk will displace a volume of fluid equal to $v \mathrm{t} \pi \mathrm{Z}^{2}$, which will induce a velocity profile at the disk edge:

$$
\begin{aligned}
& 2 \pi R t \int_{-d / 2}^{d / 2} u d z=v t \pi R^{2} \\
& \int_{-d / 2}^{d / 2} u d z=\frac{v R}{2}
\end{aligned}
$$

Integration of S.2 with respect to $\mathrm{r}$ and substitution into S.3 gives the following expression for $\Delta$ p:

$$
\Delta p=\frac{12 R^{2} \eta v}{d^{3}}
$$

The pressure term can be eliminated by substituting S.4 into S.2:

$$
u=\frac{3 R v\left(d^{2}-4 z^{2}\right)}{4 d^{3}}
$$

The maximum velocity occurs at $r=0$, the axial line midway between the two disks.

$$
u_{\max }=\frac{3 v R}{4 d}(\mathrm{~S} .6)
$$

The length of an electrode in SECM terminology is $\mathrm{R}=\mathrm{RG}^{*} \mathrm{a}$ where a is the electrode radius. Further, distances are typically normalized by the electrode radius, $\mathrm{L}=\mathrm{d} / \mathrm{a}$. Substituting into $\mathrm{S}$.6, we obtain a $v_{\max }$ that is described using SECM variables: 
$u_{\max }=\frac{3}{4} \frac{v R G}{L}(\mathrm{~S} .7)$

An analogous term can be formulated for the average velocity exiting the gap:

$\bar{u}=\frac{1}{d} \int_{-d / 2}^{d / 2} \frac{3 R v\left(d^{2}-4 z^{2}\right)}{4 d^{3}}=\frac{1}{2} \frac{v R G}{L}(\mathrm{~S} .8)$

Finally, shear is the velocity gradient normal to the disk surface:

$\dot{\gamma}=\frac{d u}{d r}=-\frac{6 z R v}{d^{3}}(\mathrm{~S} .9)$ 


\section{Derivation of Pe- $v$ relationship for a non-Newtonian fluid}

The viscosity of a non-Newtonian fluid can be described in terms of a power-law expression:

$\eta=m \dot{\gamma}^{n-1}(\mathrm{~S} .10)$

The Stokes-Einstein equation relates viscosity to a solute diffusion coefficient:

$\eta=\frac{k_{b} T}{4 \pi R^{0} D}(\mathrm{~S} .11)$

where $\mathrm{k}_{\mathrm{b}}$ is the Boltzmann constant, $\mathrm{T}$ is temperature and $\mathrm{R}^{\circ}$ is the effective solute radius. Substituting S.11 into S.10 gives:

$\frac{k_{b} T}{4 \pi R^{0}} \times \frac{1}{D}=m \dot{\gamma}^{(n-1)}$

Multiply S.12 by av and substitute the Peclect number, Pe=av/D:

$\frac{k_{b} T}{4 \pi R^{0}} \times P e=m a v \dot{\gamma}^{(n-1)}($

Assuming that the diffusion coefficient is most relevant at the edge of the electrode radius, substitute $\mathrm{R}=-\mathrm{d} / 2$ and $\mathrm{Z}=\mathrm{a}$ into equation $\mathrm{S} .9$ :

$\dot{\gamma}=\frac{3 a v}{d^{2}}(\mathrm{~S} .14)$
$\frac{k_{b} T}{4 \pi R^{0}} \times P e=\operatorname{mav}\left(\frac{3 a v}{d^{2}}\right)^{(n-1)}$

Rearrange S.15 and take the log of both sides to obtain the linearized relationship between Pe and $v$ for a nonNewtonian fluid.

$\log (P e)=n \log (v)+\log \left(\frac{4 \pi R^{\circ} m a^{n}}{k_{b} T} \times\left(\frac{3}{d^{2}}\right)^{n-1}\right)$

Equation S.16 is equation 18 in the text. 


\section{Chronoamperometry of ferrocene in ChCI:TFA DES}

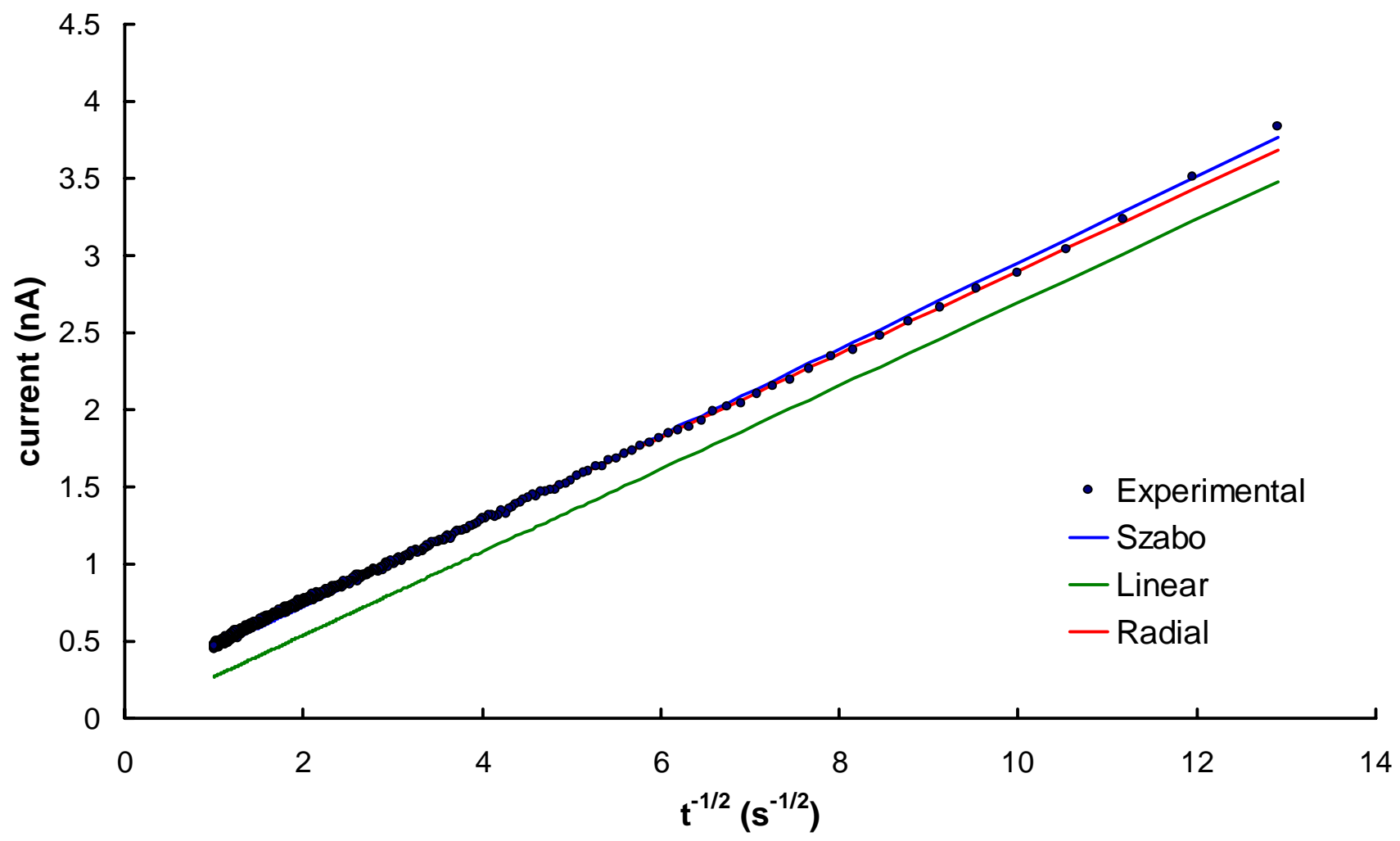

Figure S-1. Chronoamperometry of ferrocene in 1:2 ChCl:TFA DES at a $5 \mu \mathrm{m}$ radius Pt electrode. Black dots are experimental data and colored lines are best fits based on three equations as described in the text. 


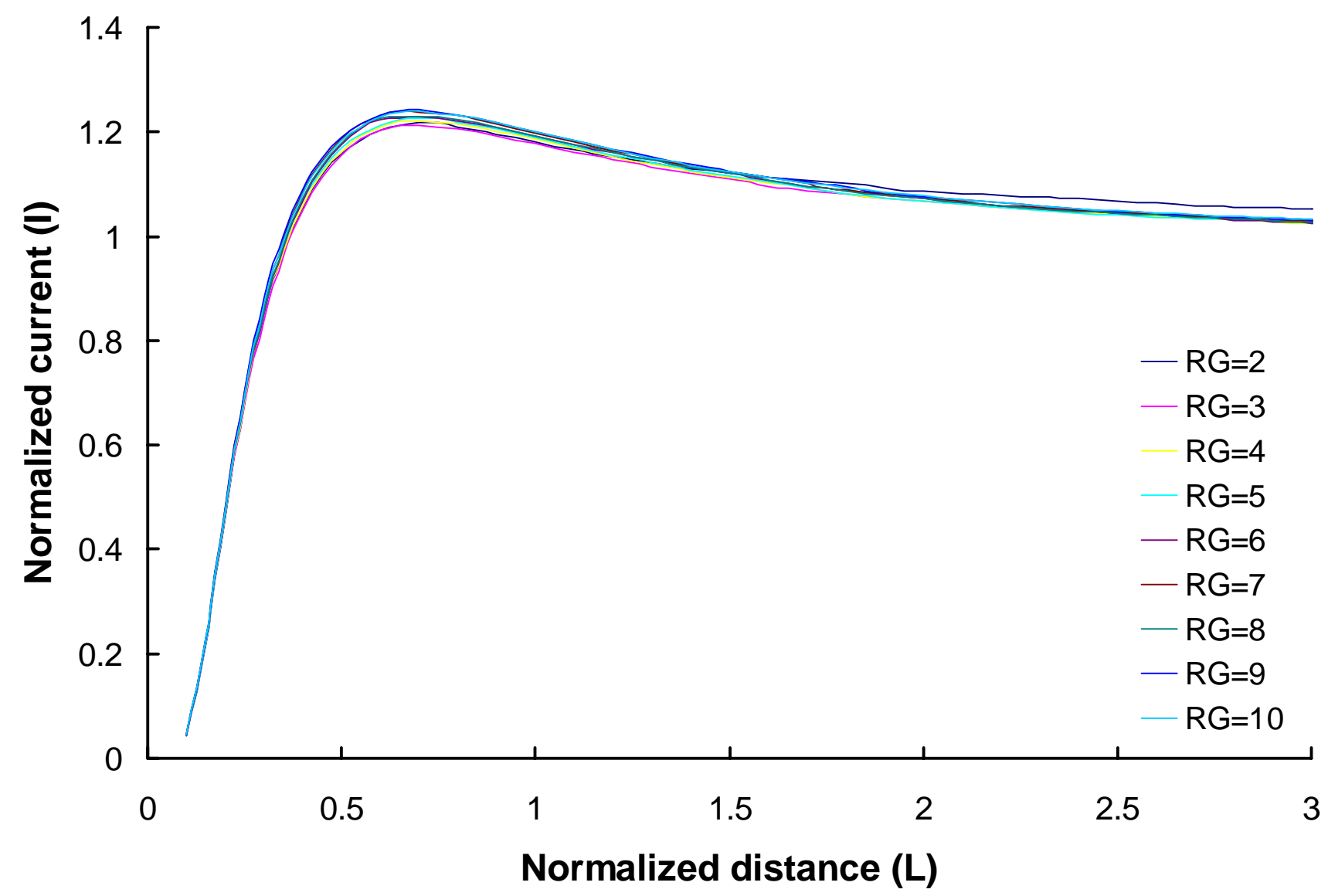

Figure S-2. Simulation of tip-velocity dependent approach curves $(\mathrm{Pe}=2)$ with varying $\mathrm{RG}$. 


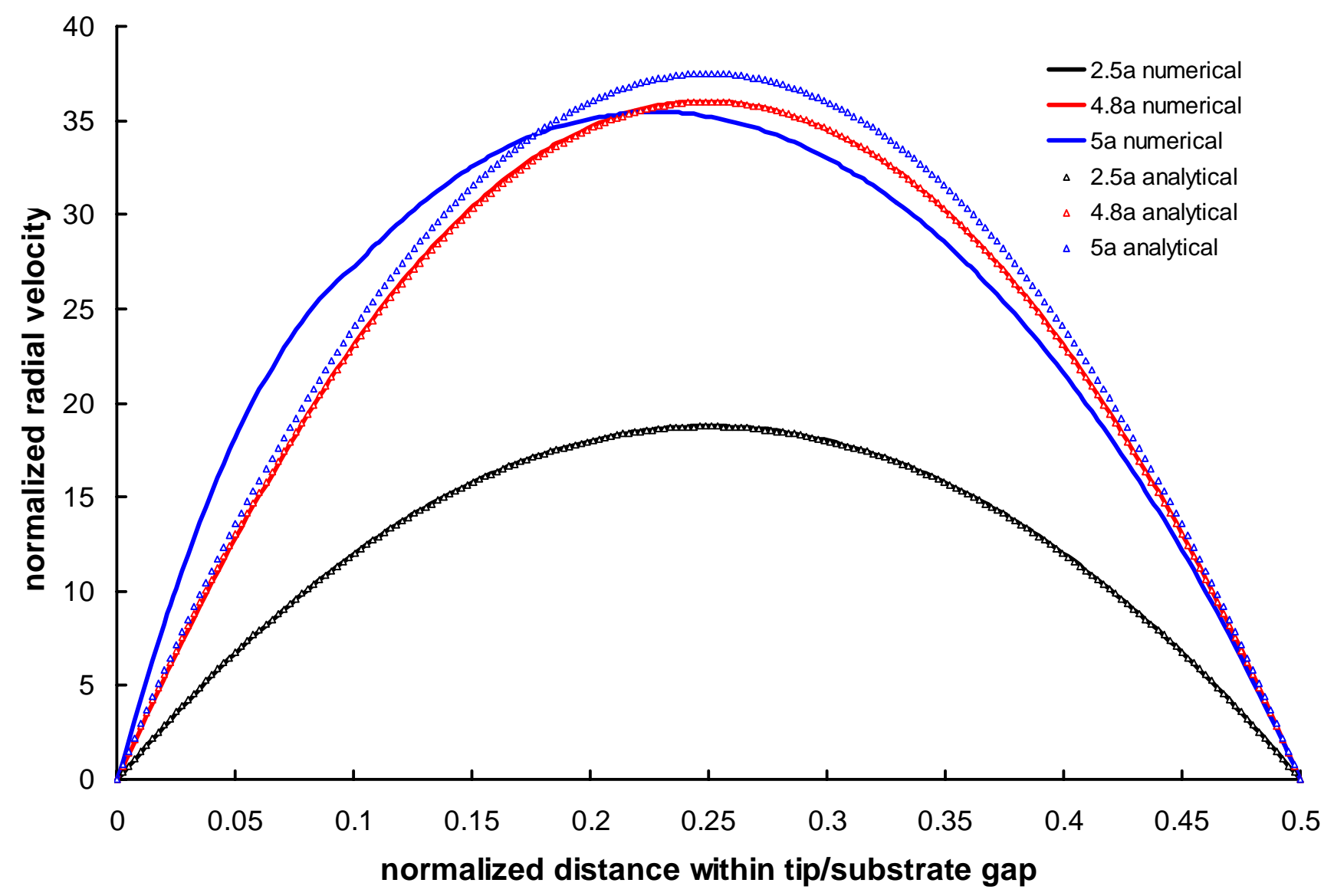

Figure S-3. Comparison of numerical and analytical solutions to the radial fluid velocity within the tip/substrate gap. Simulation parameters: $\mathrm{Pe}=5 ; \mathrm{L}=0.5 ; \mathrm{RG}=5$. The $\mathrm{x}$-axis is the distance from the tip normalized by the tip radius. 


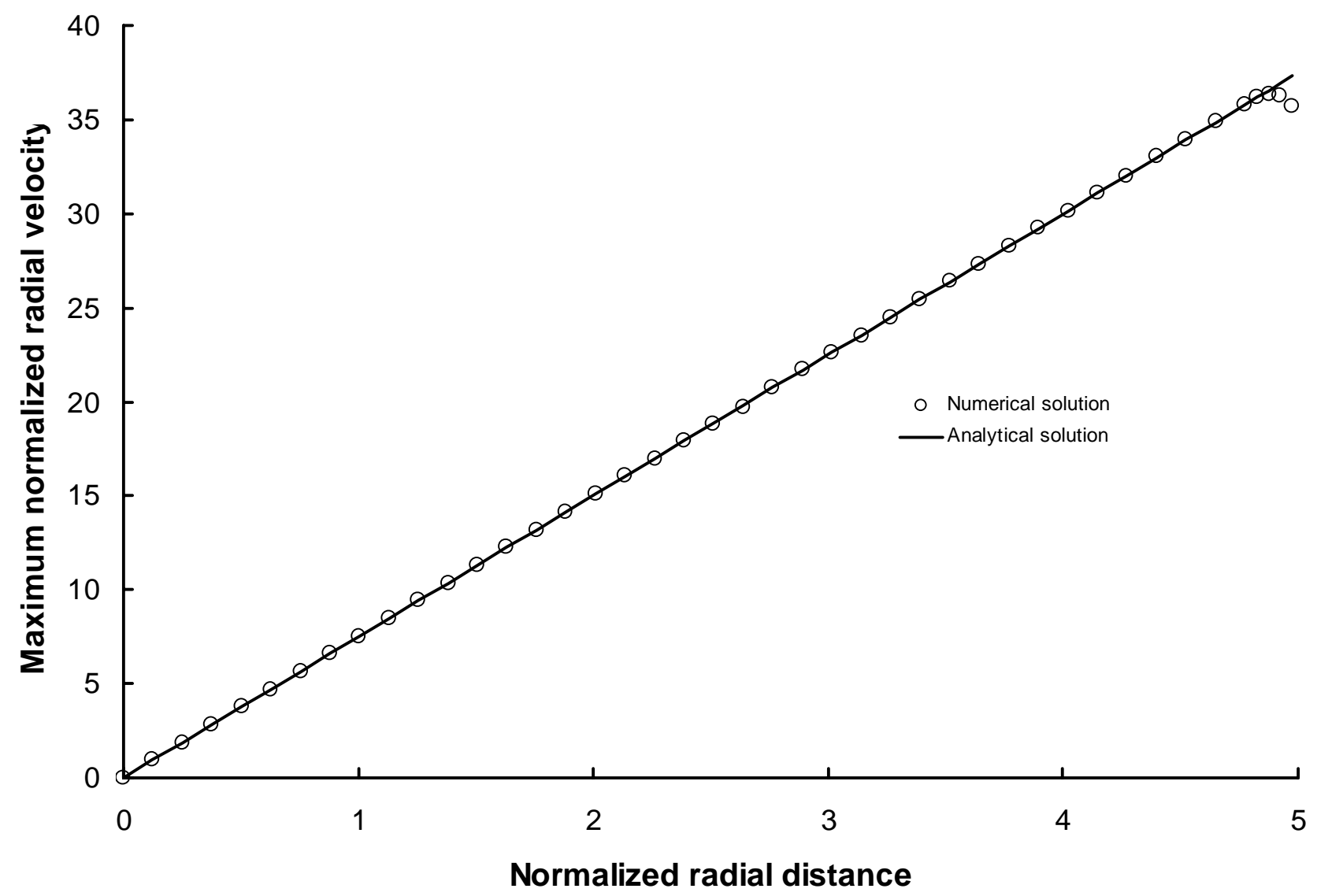

Figure S4. Comparison of maximum radial fluid velocities calculated using the numerical (COMSOL) and analytical (equation S.5) methods. Simulation parameters are identical to those in Figure S3. 


\section{COMSOL Script used to generate tip-velocity dependent insulator approach curves}

The code below can be cut and paste into a separate file with a '.m' extension in order to generate a series of tip-velocity dependent insulator approach curves. As written, the code will generate 5 separate approach curves for PE values from 1 to 5. The final approach curve is stored in an excel file. Each approach curve simulation is divided into a series of COMSOL model files. The number of files depends on the deformed mesh quality criterion (variable MAXALE). Below are a few comments for COMSOL users who wish to use this script immediately. The authors are more than willing to discuss script specifics via email.

- The section, SECM variables, contains the standard electrochemistry variables. The variable QUIET is not implemented in this version, however can be used to set the initial value after a given quiet time as opposed to steady state.

- It is possible to obtain approach curves for non-integer PE values, however the model files will not have the .mph extension due to the way the file is named. Once the extension is added manually, the .mph file can be opened and manipulated using COMSOL.

- To perform approach curves to a conductive substrate, change the two bnd.ind lines - one each in application modes 2 and 4 - to the array noted after the comment.

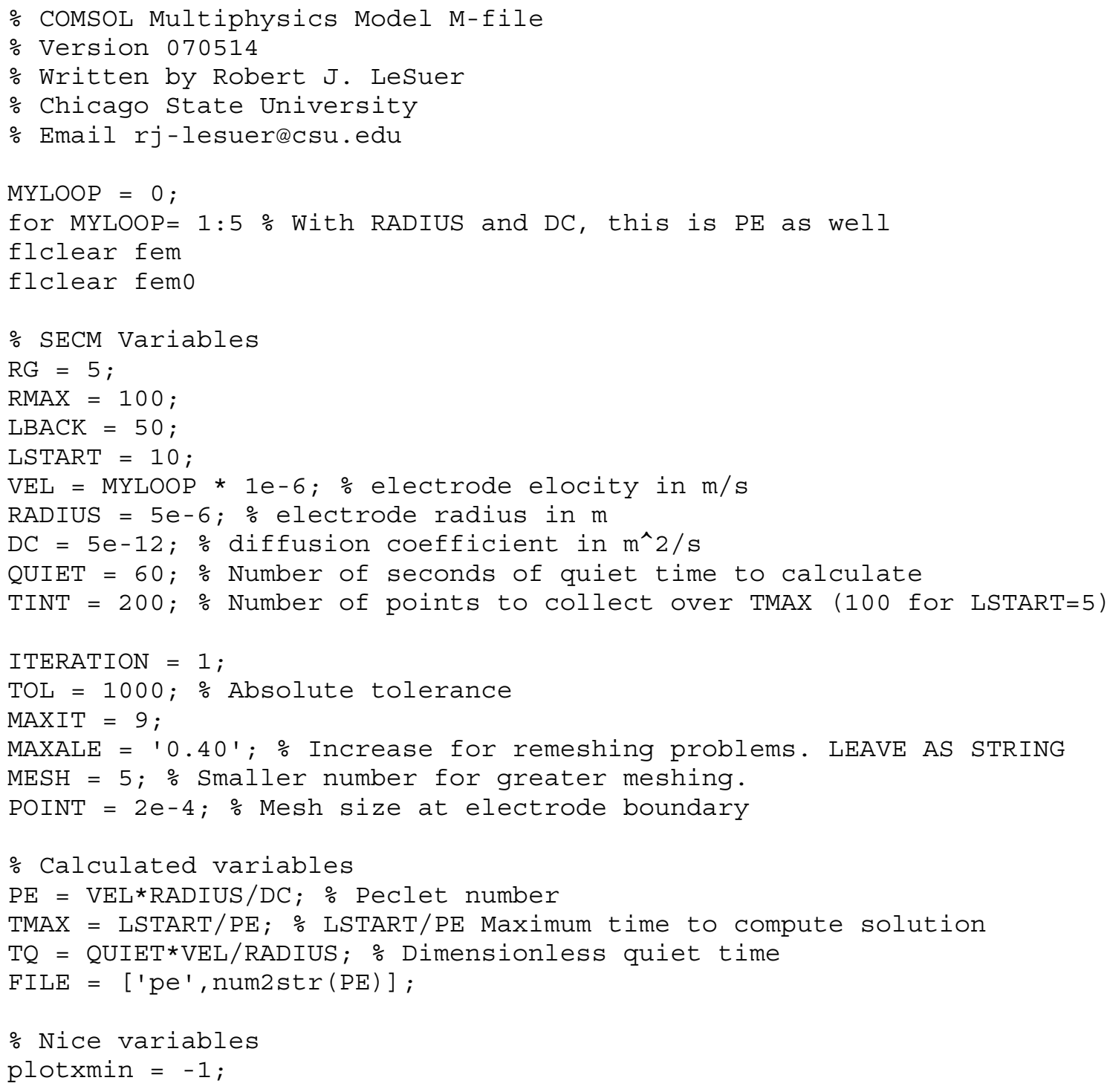


plotxmax $=1.2 * R G ;$

plotymin $=-\operatorname{LSTART} * 1.2$;

plotymax $=1$;

STOP $=($ LSTART-0.105)/PE; №rmally, (LSTART-0.2)/PE but may be changed for picky conditions

\% Introduction material

disp(['Saving simulation of $P E=$ ', num2str(PE),' to fileset ',FILE,'.'])

disp(['Will stop at ', num2str(STOP),'.'])

\% COMSOL version

clear vrsn

vrsn. name $={ }^{\prime}$ COMSOL $3.3 '$;

vrsn.ext $=1$ ';

vrsn.major $=0$;

vrsn.build $=405$;

vrsn.rCs = '\$Name: \$';

vrsn. date = '\$Date: $2006 / 08 / 31$ 18:03:47 \$';

fem.version = vrsn;

Constants

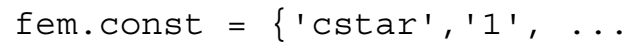

'pestat', num2str $(\mathrm{PE}), \ldots$

'restat', '1e-4', ...

'gamma', '1'\};

\% Geometry

g1=rect2 (num2str (RMAX), num2str (LBACK+LSTART), 'base ', 'corner', 'pos ', \{ '0 ', num2str (-LBACKLSTART) \}, 'rot', 'O') ;

g2=rect2 (num2str (RG), num2str (LBACK), 'base' , 'corner', 'pos ', \{ ' 0 ', num2str(-LBACKLSTART) \}, 'rot', '0');

g3 = geomcomp $\left(\{g 1, g 2\}, ' n s ',\left\{g^{\prime}{ }^{\prime}\right.\right.$, 'g2' $\}$, 'sf', 'g1-g2', 'edge', 'none' $)$;

g4=curve2 ( [RG, RMAX], [-LSTART, -LSTART $])$;

g5=curve2 ( [RG, RG], [-LSTART, 0 ] ) ;

parr $=\{$ point2 $(1,-$ LSTART $)\}$;

g7=geomcoerce ('point', parr);

\% Geometry objects

clear $\mathrm{p}$ c s

$\mathrm{p} . \mathrm{obj} \mathrm{s}=\{\mathrm{g} 7\}$;

$\mathrm{p} \cdot \mathrm{name}=\left\{\right.$ 'PT1 $\left.^{\prime}\right\}$;

$\mathrm{p} \cdot \operatorname{tags}=\left\{\mathrm{Ig}^{\prime}\right\}$;

C. obj $s=\{94,95\}$;

c. name $=\left\{\right.$ 'B1 $^{\prime}$, 'B2 $\left.^{\prime}\right\}$;

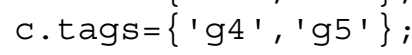

s.objs $=\left\{g^{3}\right\}$;

s. name $=\left\{\right.$ ' $\left.\mathrm{CO} 1^{\prime}\right\}$;

s. tags $=\left\{\lg ^{\prime}\right.$ ' $\}$;

fem.draw =struct ( 'p', p, 'c ', c, 's', s) ;

fem.geom $=$ geomcsg $($ fem $)$;

\% Initialize mesh

fem.mesh=meshinit (fem, 'hauto', MESH, 'hmaxvtx' , [3, POINT] ) ;

\% Application mode 1

clear appl

appl.mode.class = 'MovingMesh';

appl.mode.type = 'axi'; 


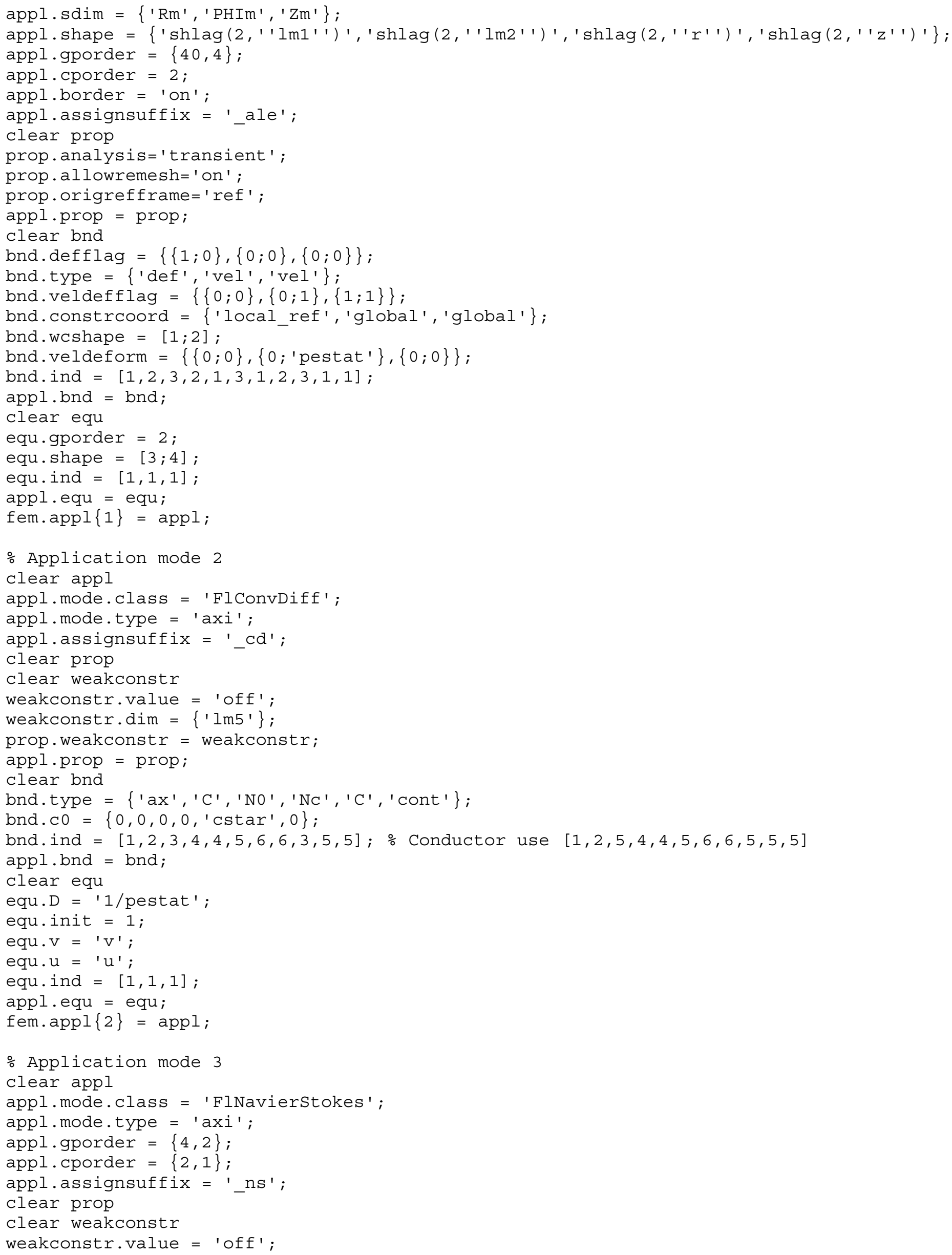




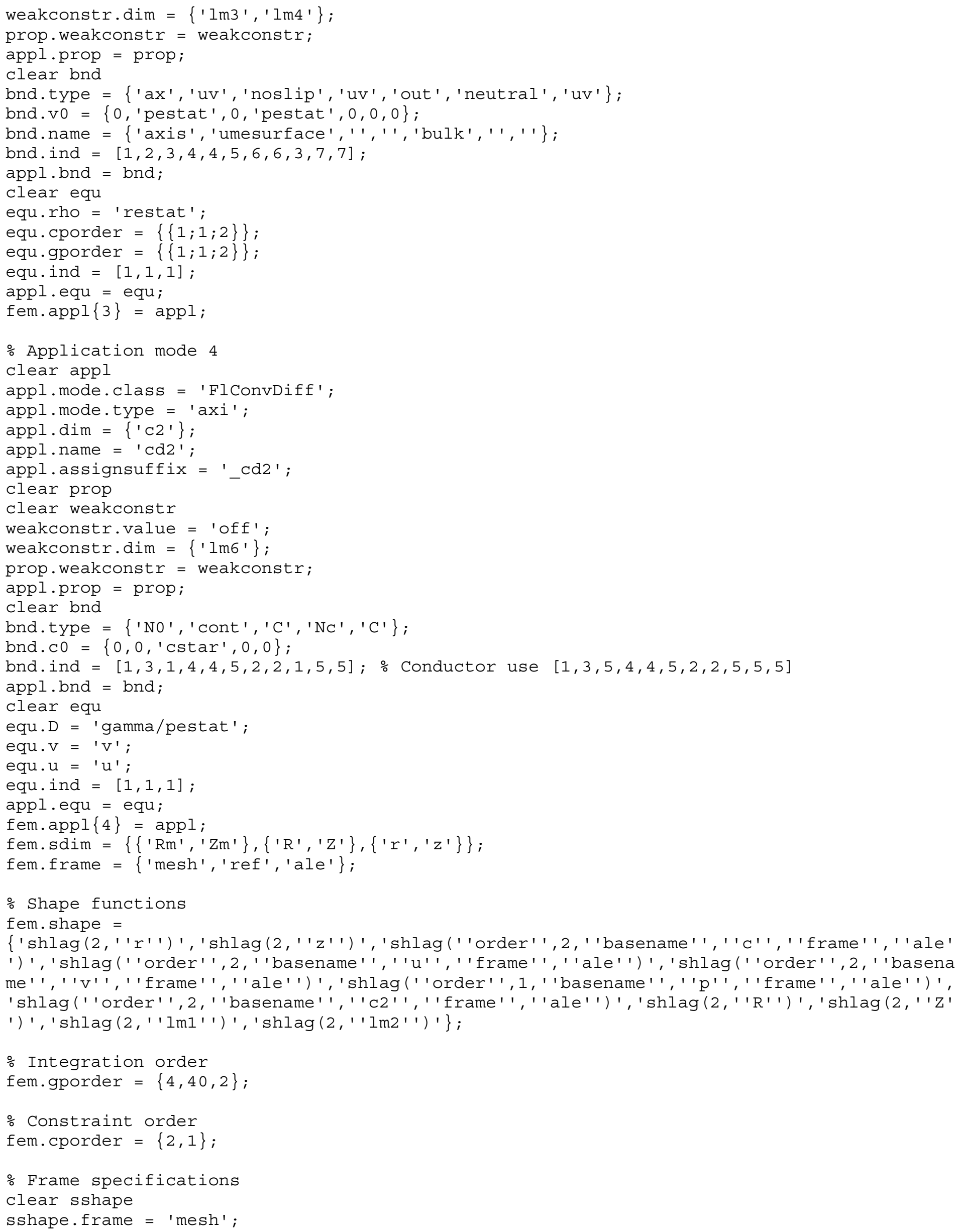




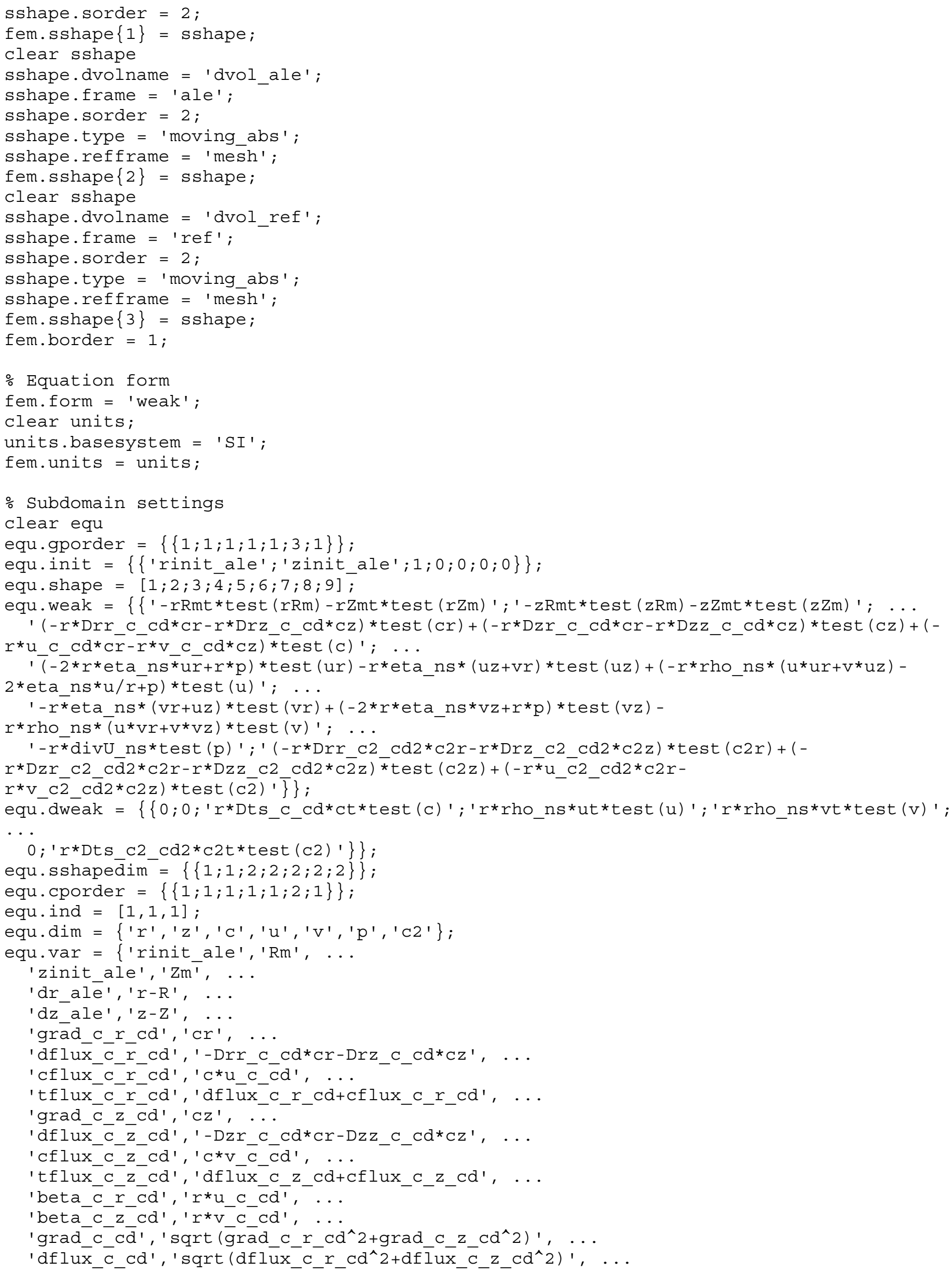


'cflux_c_cd', 'sqrt (cflux_c_r_cd^2+cflux_c_z_cd^2) ', ...

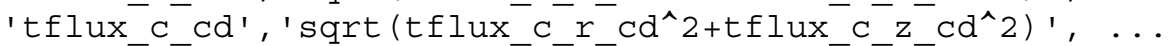

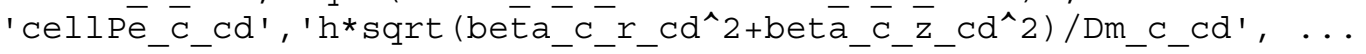

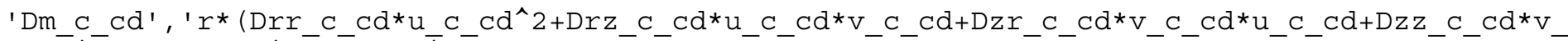
c_c $\left.\bar{d}^{\wedge} \overline{2}\right) /\left(u_{-} c_{-} c d^{\wedge} 2 \overline{+} v_{-} c_{-} c d^{\wedge} 2 \overline{t e p s}\right)^{\prime}, \ldots$

'res_c_cod', 'r*(-Drrr_c_cd*Crr-Drz_c_cd*Crz+cr*u_c_cd-Dzr_c_cd*czr-

Dzz_c_c $\left.\left.\bar{d} \overline{ }^{*} \mathrm{Czz}+\mathrm{Cz}{ }^{*} \mathrm{~V}_{-} \mathrm{C}_{-} \mathrm{c} \overline{\mathrm{d}}-\overline{\mathrm{R}}{ }_{-} \mathrm{C}_{-} \mathrm{cd}\right)\right)^{\prime}, \ldots$

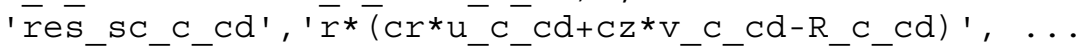

'da_c_co,', 'r*Dts_c_cd', ...

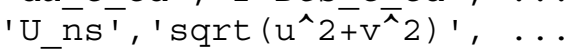

'v'ns', 'uz-vr', ...

'dīvU_ns', 'ur+vz+u/r', ...

'cellēRe_ns', 'rho_ns*U_ns*h_ale/eta_ns', ...

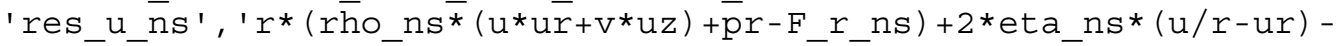

eta_ns* $(\overline{2} * r * u r r+r *(u \bar{z} z+v r z)) ', \ldots$

'res_tst_u_ns', 'r* (nojac (rho_ns)* (nojac $(u) * u r+n o j a c(v) * u z)+p r)+2 *$ nojac (eta_ns) * (u/r-

ur) - nojac (eta_ns) * $(2 * r * u r r+r *(\bar{u} z z+v r z)) ', \ldots$

'res_sc_u_ns' ' 'r* (rho_ns* $\left.(u * u r+v * u z)+p r-F \_r \_n s\right)+2 *$ eta_ns* $(u / r-u r) ', \ldots$

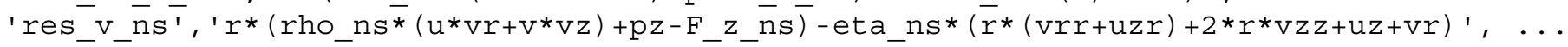

'res_tst_v_ns', 'r* (nojac (rho_ns)*(nojac $(\bar{u}) * v r+n o j a c(v) * v z)+p z)-$

nojac (eta_ns $) *\left(r *(\operatorname{vrr}+\mathrm{uzr})+2 * r \overline{\mathrm{e}}^{*} \mathrm{vzz}+\mathrm{uz}+\mathrm{vr}\right)$ ', ...

'res_sc_v_ns', 'r*(rho_ns* $\left.(\mathrm{u} * \mathrm{vr}+\mathrm{v} * \mathrm{vz})+\mathrm{pz}-\mathrm{F} \_\mathrm{z} \_\mathrm{ns}\right)$ ' , ..

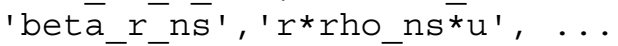

'beta_z_ns', 'r*rho_ns*v', ...

'Dm_ns' ${ }^{\prime},{ }^{\prime} r * e t a \_n s^{\prime}, \ldots$

'da_ns','r*rho_ns', ...

'gräd_c2_r_cd2', 'c2r', ...

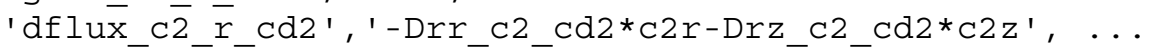

'cflux_c2_r_cd2', 'c2*u_c2_cd2', ...

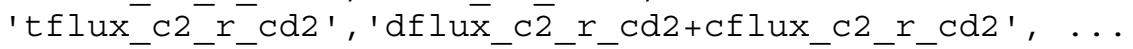

'grad_c $22_{-} \bar{z}_{-} \bar{c} d 2$ ', 'c2 $\mathrm{z}^{\prime}, \ldots$

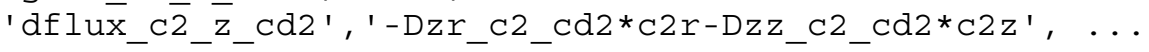

'cflux_c2_z_cd2', 'c2*V_c2 2 cd2', ...

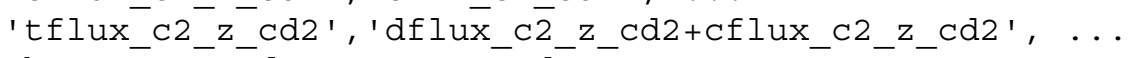

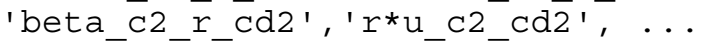

'beta_c2 ${ }^{-}{ }^{-} \mathrm{cd} 22^{\prime}, \mathrm{r}^{*} \mathrm{v}-\mathrm{c} 2{ }^{-} \mathrm{cd} 22^{\prime}, \ldots$

'grad_c2 ${ }_{-}{ }^{2} \bar{d} 22^{\prime}, ' \operatorname{sqrt}\left(\bar{g} r a \bar{d} \_c 2 \_r \_c d 2^{\wedge} 2+g r a d \_c 2 \_z \_c d 2^{\wedge} 2\right)$ '， ...

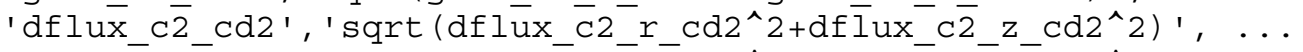

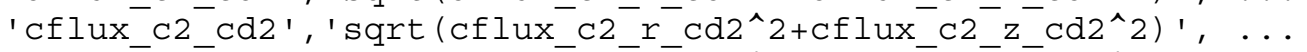

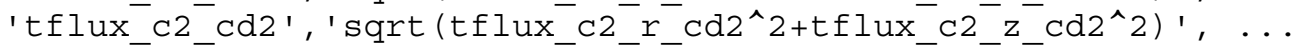

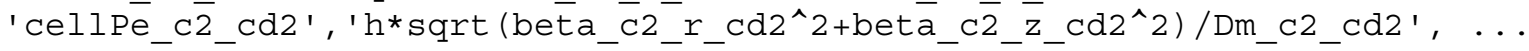

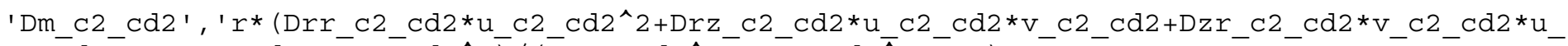

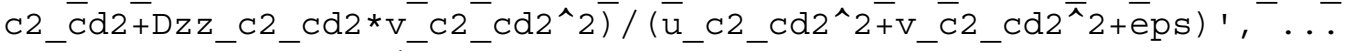

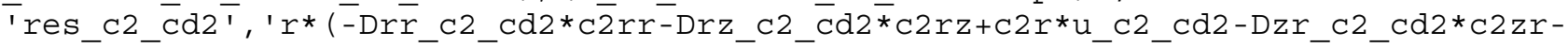

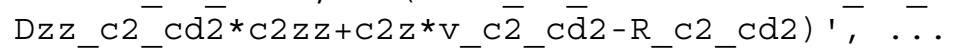

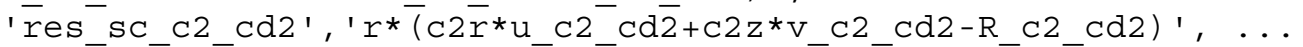

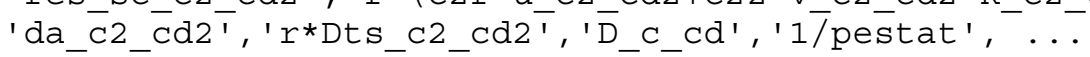

'Drēr_c_cd', '1/pestāt', ...

'Dzr_c_cd', 0, ...

'Drz_c_cd', $0, \ldots$

'Dzz_c_cd', '1/pestat', ...

'Dts_c_cd', 1, ...

'u_c_cō', 'u', ...

'v_c_cd', 'v', ...

'R_c_cd', $0, \ldots$

'rho_ns', 'restat', ...

'eta-ns', $1, \ldots$.

'F_r_ns', $0, \ldots$.

'F_z_ns', $0, \ldots$

'F_phi_ns', $0, \ldots$ 
'D_c2_cd2','gamma/pestat ', ...

'Drrr_č 2 cd2', 'gamma/pestat ', ...

'Dzr_c2_cd2',0, ...

'Drz_c2_cd2',0, ...

'Dzz_c2_cd2','gamma/pestat ', ...

'Dts_c2_cd2',1, ...

'u_c $\overline{2} \_c \bar{d} 2$ ', 'u' , ...

'v_c2_cd2', 'v', ...

'R_c2_cd2',0\};

$\mathrm{fem} \cdot$ equ $=$ equ;

\% Coupling variable elements

clear elemcpl

\% Integration coupling variables

clear elem

elem.elem = 'elcplscalar';

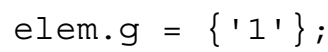

$\operatorname{src}=\operatorname{cell}(1,1)$;

clear bnd

bnd.expr $=\left\{\left\{\{\},{ }^{\prime}\right.\right.$ ndflux_c_cd', \{\}$\},\left\{\{\},{ }^{\prime} 2 *\right.$ pi*r*ntflux_c_cd', \{\}$\left.\}\right\}$;

bnd.ipoints $\left.=\left\{\left\{\{\},{ }^{\prime} 4^{\prime}, \overline{\{}\right\}\right\},\left\{\{\}, 4^{\prime},\{\}\right\}\right\}$;

bnd.frame $=\{\{\{\}$, 'ref', 'mesh' $\},\{\{\}, '$ 'ref' ', 'mesh' $\}\}$;

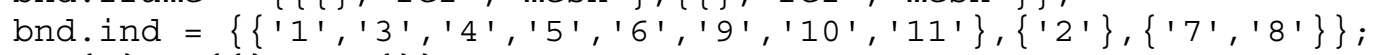

$\operatorname{src}\{1\}=\{\{\}$, bnd,\{\}$\}$;

elem.src = src;

geomdim $=\operatorname{cell}(1,1)$;

geomdim $\{1\}=\{\}$;

elem.geomdim = geomdim;

elem. var $=\{$ 'current', 'current $2 '\}$;

elem.global $=\left\{1^{\prime},{ }^{\prime} 2^{\prime}\right\}$;

elem.maxvars $=\{\}$;

elemcpl $\{1\}=$ elem;

fem.elemcpl = elemcpl;

\% Global expressions

fem.globalexpr $=\{$ 'Pe', 'velocity*a/dc','Re','velocity*dens*a/visc', 'tmax', '5/Pe'\};

- Descriptions

clear descr

descr.globalexpr $=\{$ 'Pe', 'Peclet number','Re', 'Reynolds number', 'tmax', 'tau for travelling $\mathrm{L}=5$ ' $\}$;

fem.descr $=$ descr;

․ Multiphysics

fem=multiphysics (fem, ...

'sdl', [] );

\% Extend mesh

fem.xmesh=meshextend (fem);

- Solve the steady state problem to obtain reasonable initial value

fem.sol=femstatic (fem, 'solcomp', $\left\{\right.$ 'C' $\left.^{\prime}\right\}$, 'outcomp', ...

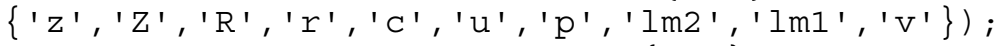

\%fem.sol=femtime (fem, 'solcomp', $\left\{\right.$ ' $\left.^{\prime}{ }^{\prime}\right\}$, 'outcomp', ...

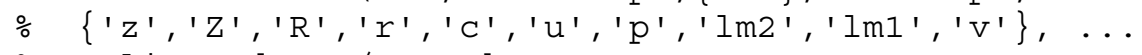

\% 'tlist', [0:TQ/10:TQ], ...

\% 'atol', \{num2str(TMAX/TOL) $\}, \ldots$

\% 'tout', 'tlist', 'tsteps', 'intermediate', ...

\% 'stopcond', ['minqual1_ale-', MAXALE] ) ;

o Save structure for restart

$\mathrm{fem} 0 \mathrm{fem}$; 


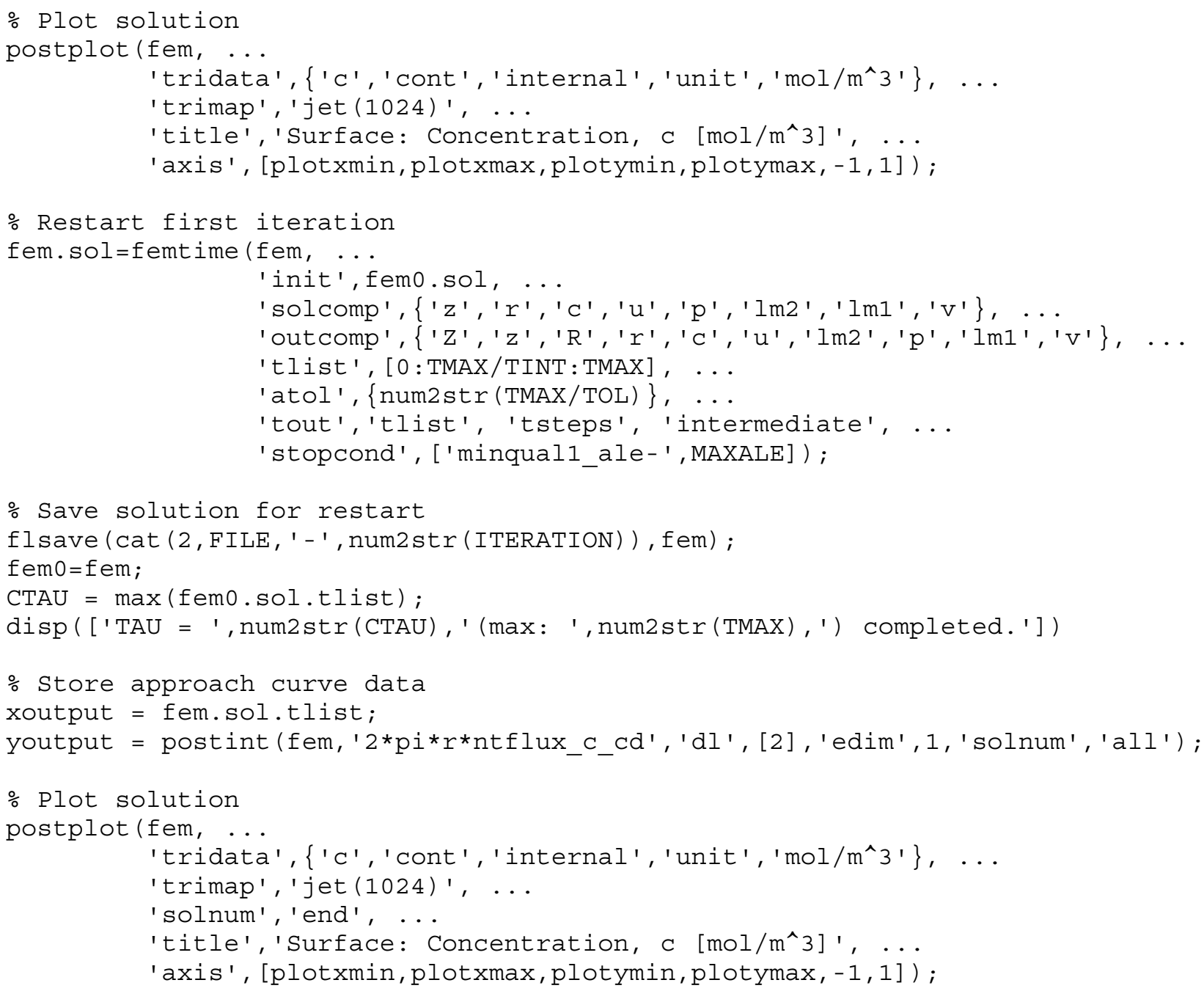

: Above will always be done, now need to iterate until at (LSTART-0.2)/PE while CTAU < STOP

\section{\% Avoid runaway scripts}

ITERATION = ITERATION + 1;

disp ( ['Iteration - ', num2str (ITERATION) ])

if ITERATION > MAXIT

break;

end

\% Lower ale stop when iterations get large

if ITERATION $==3$

$\mathrm{MESH}=5$;

POINT $=2 \mathrm{E}-4 ;$

MAXALE $=10.20$ ';

disp ( $\operatorname{MAXALE}=0.20$ and MESH=5.' )

end

\% Generate geometry from mesh

fem=mesh2geom (fem, 'srcdata', 'deformed', 'frame', 'ale') ;

fem.mesh=meshinit (fem, 'hauto' ,MESH, 'hmaxvtx' , [3, POINT] , 'hnumedg', $\{7,5\}$ );

fem.xmesh=meshextend (fem);

\% Map current solution to extended mesh

init = asseminit (fem, 'init',femo.sol, 'xmesh',femo.xmesh, 'framesrc', ... 'ale', 'domwise', 'on');

fem.sol=femtime (fem, ...

'init', init, ...

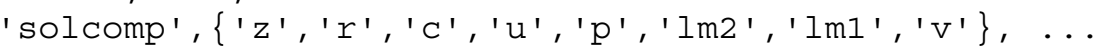

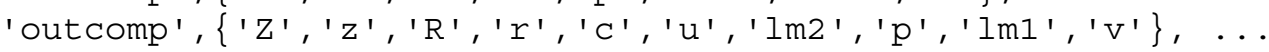




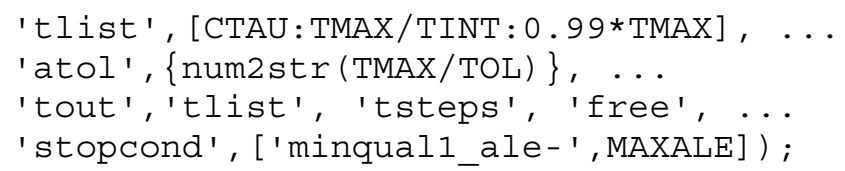

